\title{
Research on Business Performance Evaluation System for Shaanxi Listed Companies Based on EVA-BSC Coupling
}

\author{
Xiaojun Deng
}

College of Accounting and Finance, Xi'an Peihua University, Xi'an, Shaanxi

Keywords: EVA-BSC coupling; business performance evaluation; listed company; Shaanxi province

\begin{abstract}
Integrating advantages of EVA (Economic Value Adding) and BSC (Balanced Score Card), this thesis constructs a business performance evaluation system for Shaanxi listed companies and examines its application value. Based on both EVA, a financial indicator mirroring a company's value creating ability, and BSC, a non-financial but strategic indicator for performance evaluation, an empirical research on financial and performance data from 41 listed companies in Shaanxi province is conducted to examine the system's validity and practical applications. Result shows that EVA-BSC based performance ranking in 15 companies is in substantial agreement with that in EVA based raking while 26 companies see variation, demonstrating EVA-BSC based performance evaluation system's comprehensiveness and practical application value.
\end{abstract}

\section{Situation of Performance Evaluation in Shaanxi Listed Companies}

There are 46 listed companies in Shaanxi province up to the end of 2017, including entities in manufacture, finance, mining, wholesale and retail, tourism, accommodation and restaurants, typography, electric and gas and broadcast television industries. Twenty-eight manufacture companies account for 61 percent of the group, while the share of 4 mining companies is 8 percent. Three common issues in performance evaluation can be noticed after analyzing 46 companies' 2017 financial report: (1) Financial indicator is heavily weighted to maximize profits; (2) Cost of equity capital has been overlooked; (3) Non-financial indicator lacks enough attention.

\section{EVA-BSC Based Performance Evaluation System for Shaanxi Listed Companies}

According to the design principle and ideas of performance evaluation system, EVA-BSC based performance evaluation system is structured based on current operation of Shaanxi listed companies as follows(Table 1), while Standard Value of Enterprise Performance Evaluation 2016 and methods ${ }^{[1]}$ at home and abroad are referenced.

Table 1. EVA-BSC Based Performance Evaluation System for Shaanxi Listed Companies

\begin{tabular}{|c|c|c|c|}
\hline Grade I & Grade II & Calculation Formula/Meaning & Source \& Code \\
\hline \multirow{4}{*}{ Finance } & EVA & NOPAT - Cost of Capital & Model Calculation,X1 \\
\cline { 2 - 4 } & EVA Return Rate & EVA / Total Adjusted Capital & Model Calculation,X2 \\
\cline { 2 - 4 } & Return on Total Asset & Net Profit / Average Total Assets & Annual Report,X3 \\
\cline { 2 - 4 } Customer & Return On Equity & Net Profit / Owners Equity & Annual Report,X4 \\
\hline \multirow{4}{*}{ Internal Progress } & Customer Loyalty & $\begin{array}{c}\text { Top Five Customers' Revenue Proportion of } \\
\text { Operating }\end{array}$ & $\begin{array}{c}\text { Substitution } \\
\text { Variables,X5 }\end{array}$ \\
\cline { 2 - 4 } & Sales Increase & $\begin{array}{c}\text { (Current Sales - Last Year's Sales ) /Last Year's } \\
\text { Sales }\end{array}$ & Annual Report,X6 \\
\cline { 2 - 4 } & Total Asset Turnover \\
\cline { 2 - 4 } & Ratio & Net Sales / Average Total Assets & www.cninfo.com.cn,X7 \\
\cline { 2 - 4 } & Ratio of Profits to Cost & Rannual Cost of Sales / Average Stock Balance & www.cninfo.com.cn,X8 \\
\cline { 2 - 4 } & R\&D Input & Total Profit / Total Cost & Annual Report,X9 \\
\hline
\end{tabular}




\begin{tabular}{|c|c|c|c|}
\hline \multicolumn{4}{|c|}{ Cont. to Table 1} \\
\hline \multirow{3}{*}{$\begin{array}{l}\text { Learning and } \\
\text { Growth }\end{array}$} & Employee Quality & $\begin{array}{c}\text { College(and above) Educated Employees / Total } \\
\text { Employees }\end{array}$ & Annual Report,X11 \\
\hline & R\&D Ratio & R\&D Employees / Total Employees & Annual Report,X12 \\
\hline & $\begin{array}{l}\text { Per-capita Education } \\
\text { Spending }\end{array}$ & Educational Expenditure / Total Employees & Annual Report,X13 \\
\hline
\end{tabular}

Note: Due to objective reasons including considerable empirical sample, complicated data processing and inaccessible data, this thesis brings in top five customers' revenue proportion of operating in place of customer satisfaction.

\section{Empirical Examination on EVA-BSC Based Performance Evaluation System for Shaanxi Listed Companies}

\subsection{Sample selection and data sources}

Forty-one Shaanxi listed companies are selected from 46 in Shanghai and Shenzhen Stock Exchanges, getting rid of three undesirable ones and two (XINGHUA CHEMISTRY and HST) facing withdrawal risks. Disclosed data of 41 listed companies in 2107 are mainly required from audited Annual Report 2016 and 2017 published on www.cninfo.com.cn. SPSS22.0 serves for data statistics and analysis.

\subsection{EVA calculation}

Rules for Assessing Economic Value Added, published by State-owned Assets Supervision and Administration Commission (SASAC) in 2016, shows: EVA = Net Operating Profit After Tax - Capital Cost $=$ Net Operating Profit After Tax - Total Capital*Weighted Average Cost of Capital $=$ NOPAT TC*WACC.

The equation above demonstrates that EVA can be got after necessary adjustment on conventional accounting profit. Various industries, company scales and other factors require following transformation of EVA in the 41 listed companies:

Net Operating Profit after Tax $=$ Net Profit + (Interest Expense + R\&D Cost Adjustment + Depreciation reserves)*(1-25\%) + Increase in Deferred Income Tax Liabilities + Decrease in Deferred Income Tax Assets

Adjusted Total Capital $=$ Average Equity + Average Total Liabilities - Average Interest-free Current Liabilities - Average Construction in Progress + Impairment Allowance Adjustment

Weighted Average Cost of Capital Rate $=($ Cost Ratio of Equity Capital * Proportion of Equity Capital in Total Capital) + (Cost of Debt* Asset-liability Ratio)*(1- Income Tax Rate)

The cost of debt is got by taking a weighted average of interest rates of short term and long term. And cost ratio of equity capital can be worked out based on Capital Asset Pricing Model (CAPM): Cost Ratio of Equity Capital $=$ Risk-free Return Rate $+\beta^{*}$ Market Risk Premium

On the basis of models above, EVA of sample companies is exhibited in Table 2.

Table 2. EVA and EVA Return Rate of 41 Shaanxi Listed Companies

\begin{tabular}{|c|c|c|c|c|c|}
\hline Company & NOPAT & $\begin{array}{c}\text { Adjusted Total } \\
\text { Capital }\end{array}$ & WACC & EVA & $\begin{array}{c}\text { EVA Return } \\
\text { Rate }\end{array}$ \\
\hline LONGI & 2256779251.96 & 9792413583.89 & $5.26 \%$ & 1741729870.67 & $17.79 \%$ \\
\hline SHCI & 4719900332.17 & 59914995193.07 & $5.28 \%$ & 1558356512.96 & $2.60 \%$ \\
\hline CHINA XD & 1757340888.07 & 22200001884.03 & $5.60 \%$ & 514291744.90 & $2.32 \%$ \\
\hline CCOOP & 1857056204.69 & 26146254792.45 & $5.30 \%$ & 470862364.80 & $1.80 \%$ \\
\hline AECC AVIATION POWER & 1908769722.75 & 28037701851.63 & $5.19 \%$ & 453379941.33 & $1.62 \%$ \\
\hline CRE & 475633560.87 & 2800257434.51 & $5.26 \%$ & 328457589.11 & $11.73 \%$ \\
\hline SHAANXI GAS & 626674892.87 & 7749324125.48 & $5.23 \%$ & 221601638.53 & $2.86 \%$ \\
\hline SHAANGU & 538794534.75 & 7039387401.57 & $5.22 \%$ & 171512109.84 & $2.44 \%$ \\
\hline FENGHUO ELECTRONICS & 219165220.30 & 1301040590.55 & $5.36 \%$ & 149395368.90 & $11.48 \%$ \\
\hline
\end{tabular}




\begin{tabular}{|c|c|c|c|c|c|}
\hline \multicolumn{6}{|c|}{ Cont. to Table 2} \\
\hline YCHJ & 245997362.64 & 1899899921.01 & $5.25 \%$ & 146159948.49 & $7.69 \%$ \\
\hline HNA-CAISSA TRAVEL & 276415211.53 & 3008224841.16 & $5.13 \%$ & 122113977.85 & $4.06 \%$ \\
\hline ZEMIC & 169468879.03 & 1421147301.21 & $4.10 \%$ & 111135649.34 & $7.82 \%$ \\
\hline $\begin{array}{l}\text { CECEP ENVIRONMENTAL } \\
\text { PROTECTION EQUIPMENT }\end{array}$ & 182208825.86 & 1739836247.21 & $5.19 \%$ & 91878963.60 & $5.28 \%$ \\
\hline SHAANXI HEIMAO & 425082189.36 & 6412779742.67 & $5.21 \%$ & 90882014.56 & $1.42 \%$ \\
\hline SCMC & 256305892.03 & 3792749223.50 & $5.25 \%$ & 57142175.48 & $1.51 \%$ \\
\hline SUNRESIN NEW MATERIALS & 79545226.09 & 662820031.94 & $4.45 \%$ & 50050505.99 & $7.55 \%$ \\
\hline TYPICAL & 113679004.14 & 1323374748.95 & $5.40 \%$ & 42153770.59 & $3.19 \%$ \\
\hline CHENXI AVIATION & 61864804.48 & 374086466.80 & $5.37 \%$ & 41786526.00 & $11.17 \%$ \\
\hline BVEA & 56620481.08 & 545166983.28 & $5.31 \%$ & 27695910.87 & $5.08 \%$ \\
\hline GLOBAL PRINTING & 47340614.70 & 526147653.27 & $5.35 \%$ & 19207056.76 & $3.65 \%$ \\
\hline QJCT & 86246802.44 & 1312106241.74 & $5.32 \%$ & 16458671.67 & $1.25 \%$ \\
\hline SHAANXI JINYE & 84618383.00 & 1306127319.84 & $5.29 \%$ & 15549652.34 & $1.19 \%$ \\
\hline SXBN & 191018199.80 & 3326096912.24 & $5.30 \%$ & 14750818.66 & $0.44 \%$ \\
\hline WESTERN METAL MATERIALS & 171954861.57 & 2611001662.68 & $6.09 \%$ & 12891834.31 & $0.49 \%$ \\
\hline $\begin{array}{c}\text { NTERNATIONAL MEDICAL } \\
\text { INVESTMENT }\end{array}$ & 234290316.81 & 4276603314.48 & $5.29 \%$ & 8050788.48 & $0.19 \%$ \\
\hline TIANHE DEFENSE & 73572353.06 & 1433124283.67 & $5.34 \%$ & -3023516.06 & $-0.21 \%$ \\
\hline BUT'ONE & 7378268.69 & 225167070.68 & $5.06 \%$ & -4005118.21 & $-1.78 \%$ \\
\hline DAGANG ROAD MACHINERY & 33554992.12 & 852417925.30 & $4.71 \%$ & -6589225.29 & $-0.77 \%$ \\
\hline GINWA & 40183792.08 & 954944525.67 & $5.35 \%$ & -10869269.01 & $-1.14 \%$ \\
\hline XI'AN CATERING & 13887976.12 & 808677345.54 & $5.11 \%$ & -27472957.44 & $-3.40 \%$ \\
\hline XI'AN TOURISM & 20440613.07 & 1060401076.02 & $5.27 \%$ & -35411277.09 & $-3.34 \%$ \\
\hline J\&R OPTIMUM ENERGY & 317317307.96 & 7379813720.81 & $5.17 \%$ & -64043467.80 & $-0.87 \%$ \\
\hline TONG OIL TOOLS & 38500973.66 & 1937568313.42 & $5.42 \%$ & -66590469.59 & $-3.44 \%$ \\
\hline LIGEANCE AEROSPACE & -29463948.20 & 1365451689.54 & $4.98 \%$ & -97468823.06 & $-7.14 \%$ \\
\hline BAOTI & 234078901.25 & 6373354510.41 & $5.24 \%$ & -99951502.80 & $-1.57 \%$ \\
\hline QINCHUAN MACHINE TOOL & 180118854.76 & 5574325158.35 & $5.19 \%$ & -109276143.83 & $-1.96 \%$ \\
\hline AEROSPACE POWER & 52316802.63 & 3170752531.26 & $5.29 \%$ & -115535274.57 & $-3.64 \%$ \\
\hline BODE ENERGY EQUIPMENT & 108424811.23 & 4779765118.72 & $5.02 \%$ & -131367142.15 & $-2.75 \%$ \\
\hline AVIC AIRCRAFT & 895383742.63 & 19803884327.54 & $5.20 \%$ & -134113861.30 & $-0.68 \%$ \\
\hline CDD & -90816127.61 & 2721737134.98 & $5.10 \%$ & -229564498.43 & $-8.43 \%$ \\
\hline JDCMOLY & 99346380.46 & 12306060110.92 & $5.43 \%$ & -569148790.81 & $-4.62 \%$ \\
\hline
\end{tabular}

\subsection{Examination on Application of EVA-BSC Based Performance Evaluation System}

Factor analysis is applied to examine the feasibility and interpretability of EVA-BSC based performance evaluation system for 41 sample companies. In accordance with dimensionality reduction, several factors are brought in to replace intricate multidimensional data on the basis of their internal matrix, simplifying sample data and minimizing data loss.

3.3.1 Feasibility Examination on PCA

Before principal component analysis (PCA), KMO (Kaiser-Meyer-Olkin) and Bartlett's Test of Sphericity ${ }^{[2]}$ are conducted to examine PCA's feasibility and results are demonstrated in Table 3 , in which $\mathrm{KMO}=0.482$, closing to 0.5, and chi-square is 348.749 in Bartlett's Test of Sphericity, higher than 
the critical value (18.3) with a lower significance level of 0.05 . Information above indicates reasonable sample selection and feasible PCA.

Table 3 KMO and Bartlett's Test of Sphericity

\subsubsection{Factor Number}

\begin{tabular}{|l|l|l|}
\hline KMO & Approx. Chi-Square & 348.749 \\
\hline \multirow{3}{*}{ Bartlett's Test of Sphericity } & Df & 78 \\
\cline { 2 - 3 } & Sig. & .000 \\
\hline
\end{tabular}

Factor Number is usually in agreement with principal component number in PCA so that principal factors can accumulatively contribute to sample variance to a certain extent. Eigenvalue, eigenvalue contribute and cumulative contribute in Table 4 provides reasonable interpretation for the factor model.

Table 4. Total Variance Explained

\begin{tabular}{|l|r|r|r|r|r|r|}
\hline \multirow{2}{*}{ Factor } & \multicolumn{3}{|c|}{ Initial Eigenvalue } & \multicolumn{3}{|c|}{ Extraction Sums of Squared Loadings } \\
\cline { 2 - 7 } & Total & \% of Variance & Cumulative \% & Total & \% of Variance & Cumulative \% \\
\hline 1 & 4.151 & 31.930 & 31.930 & 4.151 & 31.930 & 31.930 \\
\hline 2 & 2.080 & 15.999 & 47.929 & 2.080 & 15.999 & 47.929 \\
\hline 3 & 1.558 & 11.983 & 59.912 & 1.558 & 11.983 & 59.912 \\
\hline 4 & 1.250 & 9.612 & 69.524 & 1.250 & 9.612 & 69.524 \\
\hline 5 & .976 & 7.510 & 77.034 & & & \\
\hline 6 & .962 & 7.399 & 84.433 & & & \\
\hline 7 & .678 & 5.214 & 89.648 & & & \\
\hline 8 & .541 & 4.158 & 93.805 & & & \\
\hline 9 & .364 & 2.802 & 96.608 & & & \\
\hline 10 & .218 & 1.673 & 98.281 & & & \\
\hline 11 & .115 & .884 & 99.165 & & & \\
\hline 12 & .100 & .769 & 99.934 & & & \\
\hline 13 & .009 & .066 & 100.000 & & & \\
\hline
\end{tabular}

Eigenvalue of Factor 1(4.151) in Table 4 explains the 31.930\% of Variance and present maximum cumulative contribute, indicating its strongest integration capability on original variables, while Factor 2 explains the $15.999 \%$ of Variance, $11.983 \%$ for Factor 3 and $9.612 \%$ for Factor 4, with approximate cumulative contribute of 70\%, almost covering 13 components adopted. Above all, 13 components can be reduced to 4 factors.

3.3.3 Factor Analysis

Unrotated factor matrix is showed in Table 5 and rotated factor matrix in Table 6. Normally, the distribution of variables is more dispersed in the factor loading after rotation, meaning better explanation ${ }^{[3]}$ so than further analysis is conducted based on the rotated factor matrix.

Table 5. Unrotated Factor Matrix ${ }^{a}$

\begin{tabular}{|l|r|r|r|r|}
\hline \multirow{2}{*}{} & \multicolumn{4}{|c|}{ Factor } \\
\cline { 2 - 5 } & 1 & \multicolumn{1}{|c|}{2} & \multicolumn{1}{c|}{3} & \multicolumn{1}{c|}{4} \\
\hline $\mathrm{X} 1$ & .531 & .243 & .147 & -.545 \\
\hline $\mathrm{X} 2$ & .778 & .514 & .028 & -.121 \\
\hline $\mathrm{X} 3$ & .851 & .403 & .061 & -.015 \\
\hline $\mathrm{X} 4$ & .856 & .294 & .134 & -.055 \\
\hline $\mathrm{X} 5$ & -.344 & .618 & .186 & .000 \\
\hline $\mathrm{X} 6$ & .085 & -.185 & -.375 & -.274 \\
\hline $\mathrm{X} 7$ & .712 & -.274 & .460 & .274 \\
\hline $\mathrm{X} 8$ & .348 & -.323 & .590 & .395 \\
\hline $\mathrm{X} 9$ & -.716 & .304 & .522 & -.225 \\
\hline $\mathrm{X} 10$ & .671 & -.205 & -.617 & .237 \\
\hline $\mathrm{X} 11$ & -.259 & .451 & -.140 & .597 \\
\hline
\end{tabular}




\begin{tabular}{|l|r|r|r|r|}
\hline \multicolumn{5}{|c|}{ Cont. to Table 5 } \\
\hline $\mathrm{X} 12$ & -.149 & .755 & -.255 & .306 \\
\hline $\mathrm{X} 13$ & -.052 & .056 & -.203 & -.270 \\
\hline
\end{tabular}

Table 6. Rotated Factor Matrix ${ }^{\mathrm{a}}$

\begin{tabular}{|l|r|r|r|r|}
\hline & \multicolumn{4}{|c|}{ Factor } \\
\cline { 2 - 5 } & \multicolumn{1}{|c|}{1} & \multicolumn{1}{|c|}{2} & \multicolumn{1}{l|}{3} & \multicolumn{1}{c|}{4} \\
\hline $\mathrm{X} 1$ & .726 & -.097 & -.303 & -.178 \\
\hline $\mathrm{X} 2$ & .919 & .144 & .137 & .020 \\
\hline $\mathrm{X} 3$ & .893 & .240 & .101 & .157 \\
\hline $\mathrm{X} 4$ & .864 & .223 & -.023 & .207 \\
\hline $\mathrm{X} 5$ & .081 & -.554 & .457 & -.115 \\
\hline $\mathrm{X} 6$ & -.011 & .281 & -.215 & -.363 \\
\hline $\mathrm{X} 7$ & .413 & .264 & -.288 & .738 \\
\hline $\mathrm{X} 8$ & .087 & .045 & -.215 & .820 \\
\hline $\mathrm{X} 9$ & -.255 & -.924 & .073 & -.059 \\
\hline $\mathrm{X} 10$ & .253 & .929 & .039 & -.032 \\
\hline $\mathrm{X} 11$ & -.164 & -.026 & .777 & .122 \\
\hline $\mathrm{X} 12$ & .146 & -.108 & .826 & -.190 \\
\hline $\mathrm{X} 13$ & .033 & -.002 & -.061 & -.340 \\
\hline
\end{tabular}

According to Table 6, Factor 1 presents high load on X1(EVA), X2(EVA Return Rate), X3(Return on Total Asset), X4(Return On Equity) and X7(Total Asset Turnover Ratio); Factor 2 presents high load on X5(Customer Loyalty), X9(R\&D Input) and X10(Ratio of Profits to Cost); Factor 3 presents high load on X1(EVA), X5(Customer Loyalty),X11(Employee Quality) and X12(R\&D Ratio); Factor 4 presents high load on X6(Sales Increase), X7(Total Asset Turnover Ratio), X8(Inventory Turnover) and X13(Per-capita Education Spending).

Further, factor score coefficient matrix, which matches factors and corresponding original variables, is showed in Table 7.

Table 7. Factor Score Coefficient Matrix

\begin{tabular}{|l|r|r|r|r|}
\hline & \multicolumn{4}{|c|}{ Factor } \\
\cline { 2 - 5 } & \multicolumn{1}{|c|}{1} & \multicolumn{1}{|c|}{2} & \multicolumn{1}{l|}{3} & \multicolumn{1}{c|}{4} \\
\hline $\mathrm{X} 1$ & .303 & -.187 & -.235 & -.217 \\
\hline $\mathrm{X} 2$ & .307 & -.045 & .076 & -.062 \\
\hline $\mathrm{X} 3$ & .272 & .000 & .082 & .025 \\
\hline $\mathrm{X} 4$ & .262 & -.021 & .011 & .048 \\
\hline $\mathrm{X} 5$ & .109 & -.243 & .196 & -.014 \\
\hline $\mathrm{X} 6$ & -.006 & .136 & -.137 & -.277 \\
\hline $\mathrm{X} 7$ & .051 & .016 & -.072 & .417 \\
\hline $\mathrm{X} 8$ & -.045 & -.045 & -.036 & .519 \\
\hline $\mathrm{X} 9$ & .047 & -.435 & -.064 & .026 \\
\hline $\mathrm{X} 10$ & -.043 & .454 & .123 & -.074 \\
\hline $\mathrm{X} 11$ & -.089 & .098 & .485 & .185 \\
\hline $\mathrm{X} 12$ & .063 & .027 & .462 & -.045 \\
\hline $\mathrm{X} 13$ & .045 & -.001 & -.076 & -.238 \\
\hline
\end{tabular}

According to Table 7, linear relationship between 4 factors and 13 indicators is as follows:

$\mathrm{Y} 1=0.303 \mathrm{X} 1+0.307 \mathrm{X} 2+0.272 \mathrm{X} 3+0.262 \mathrm{X} 4+0.109 \mathrm{X} 5-0.006 \mathrm{X} 6+0.051 \mathrm{X} 7-0.045 \mathrm{X} 8+$ $0.047 \mathrm{X} 9-0.043 \times 10-0.089 \times 11+0.063 \times 12+0.045 \times 13$

$\mathrm{Y} 2=-0.187 \mathrm{X} 1-0.045 \mathrm{X} 2+0 \mathrm{X} 3-0.021 \mathrm{X} 4-0.243 \mathrm{X} 5+0.136 \mathrm{X} 6+0.016 \mathrm{X} 7-0.045 \mathrm{X} 8-0.435 \mathrm{X} 9$ $+0.454 \mathrm{X} 10-0.098 \mathrm{X} 11+0.027 \mathrm{X} 12-0.001 \mathrm{X} 13$

$$
\mathrm{Y} 3=-0.235 \mathrm{X} 1+0.076 \mathrm{X} 2+0.082 \mathrm{X} 3+0.011 \mathrm{X} 4+0.196 \mathrm{X} 5-0.137 \mathrm{X} 6-0.072 \mathrm{X} 7-0.036 \mathrm{X} 8-
$$
$0.064 X 9+0.123 \times 10+0.485 \times 11+0.462 \times 12-0.076 \times 13$ 


$$
\mathrm{Y} 4=-0.217 \mathrm{X} 1-0.062 \mathrm{X} 2+0.025 \mathrm{X} 3+0.048 \mathrm{X} 4-0.014 \mathrm{X} 5-0.277 \mathrm{X} 6+0.417 \mathrm{X} 7+0.519 \mathrm{X} 8+
$$

\section{$0.026 \times 9-0.074 X 10+0.185 \times 11-0.045 \times 12-0.238 \times 13$}

Combining with data from 41 Shaanxi listed companies in 2017, formulas above work out factor scores, which are then weighted by variance, contribute / aggregate variance to get composite score and performance ranking for 41 companies based on formula $\mathrm{Y}=45.90 \% \mathrm{Y} 1+23.00 \% \mathrm{Y} 2+12.20 \% \mathrm{Y}+$ $13.80 \% Y 4$.

\section{Conclusion}

Result demonstrates negative EVA in 16 sample listed companies, illustrating that they get accounting profit without financial value creation. In these companies, TIANHE DEFENSE, TONG OIL TOOLS, BUT'ONE, CDD, LIGEANCE AEROSPACE, BODE ENERGY EQUIPMENT and XI'AN CATERING are in the red, while another 9 companies get positive net profit with extremely low equity capital utilization, such as JDCMOLY and AVIC AIRCRAFT, making up the deficits and getting surpluses by comprehensive income items adjustment. Meanwhile, these 16 companies are experiencing value impairment. For instance, LONGI, working on R\&D, production and sales of silicon rod, wafer and battery, bear highest EVA, which stands for increasing market share and extensive prospect. On the other side, 26 companies see ranking variation during the research, especially SXBN, GINWA, BUT'ONE, WESTERN METAL MATERIALS, LIGEANCE AEROSPACE and SCMC, which partly proves the comprehensiveness and actual value of EVA-BSC based performance evaluation system after bringing non-financial indicators into the conventional one.

\section{Reference}

[1]Mousui Modak, K hanindra Pathak, Kunal Kanti Ghosh. Performance evaluation of out sourcing decision using a BSC and Fuzzy AHP approach: A case of the Indian coalmining organization[J].Resources Policy,2017.

[2]Ollor W G, Dagogo D W. The Effect of Venture Capital Financing on the Economic Value Added Profile of Nigerian SMEs[J].Social Science Electronic Publishing,2009(5).

[3]Robert S. Kaplan, David P. Norton. Put the Balanced Scorecard into work[J].Harvard Business Review,1993 